\title{
Periprocedural anticoagulation in atrial fibrillation: Update on electrical cardioversion and ablation
}

\author{
S. P. G. van Vugt ${ }^{1}$ M. A. Brouwer ${ }^{1}$
}

Published online: 9 May 2018

(c) The Author(s) 2018

\begin{abstract}
In this manuscript, we discuss the most important changes in the field of anticoagulant treatment in patients with atrial fibrillation in the setting of electrical cardioversion or catheter ablation. Moreover, we provide practical guidance as well as information on daily practice.
\end{abstract}

Keywords Atrial fibrillation · Electrical cardioversion · Catheter ablation · Vitamin K antagonist · Non-vitamin K oral anticoagulant

\section{Electrical cardioversion for atrial fibrillation}

For successful restoration of sinus rhythm in patients with atrial fibrillation (AF), electrical cardioversion (ECV) is a quicker and more effective strategy than pharmacological cardioversion, with the highest success rates in case of pretreatment with antiarrhythmic drugs [1-5]. However, the success rate should be weighed against the involved disadvantage of sedation, the need of fasting, and the procedurerelated increase in stroke risk [6].

After the landmark AF trials with the non-vitamin K oral anticoagulants (NOACs), several randomised trials on ECV have been published [7-9]. The periprocedural use of the new agents is now addressed in more detail in the $2016 \mathrm{Eu}-$ ropean $\mathrm{AF}$ guidelines [3]. For patients in whom cardioversion is performed within 48 hours of AF onset, the recommendations with regard to periprocedural anticoagulation have become less liberal over the years [3, 10]. Notably, preprocedural anticoagulation is now recommended for all patients, irrespective of risk factors for stroke. The same holds true for the 4-week postprocedural anticoagulation regimen. These recommendations pertain to both chemical and electrical cardioversion [3].

\footnotetext{
S. P. G. van Vugt

S.vanVugt@radboudumc.nl

M. A. Brouwer

Marc.Brouwer@radboudumc.nl

1 Department of Cardiology, Radboud University Medical Center, Nijmegen, The Netherlands
}

In addition to a short update of the most important changes, and a review of the NOAC-related regimens, we provide some practical guidance and information on daily practice of anticoagulation in the setting of cardioversion in Europe.

\section{The evidence}

\section{Periprocedural anticoagulation}

Whereas for AF of more than 48 hours of duration no important changes have been reported, a stricter regimen has been introduced for anticoagulation therapy prior to ECV in the setting of short duration AF (Fig. 1; [3]). Previously, patients with $\mathrm{AF}<48$ hours were triaged for preprocedural heparin based on their stroke risk profile, but the large FinCV registry on 5116 cardioversions reported a clear increase in the risk of stroke when AF duration exceeded 12 hours. This also applied to patients with $\mathrm{CHA}_{2} \mathrm{DS}_{2}-$ VASc [Congestive heart failure, Hypertension, Age $\geq 75$ [doubled], Diabetes, prior Stroke [doubled]—Vascular disease, Age 65-74, Sex category] scores of 0-1 (Table 1; [11]). In the subset of 2,481 patients with neither preprocedural heparin nor subsequent anticoagulation therapy, the risk of thrombo-embolic events for $\mathrm{CHA}_{2} \mathrm{DS}_{2}$-VASc $0-1$ was at least as high as for patients with $\mathrm{CHA}_{2} \mathrm{DS}_{2}$-VASc 2 [12]. Stroke rates were markedly lower when on antithrombotic therapy, with no events in $\mathrm{CHA}_{2} \mathrm{DS}_{2}$-VASc $0-1$ in those on treatment versus $0.4 \%$ to those without [13]. Whereas in the 2010 European AF guideline preprocedural heparin was recommended based upon the estimated risk profile for stroke (Class I-B), the latest guidelines advo- 


\section{KEY MESSAGE}

- Prior to electrical/pharmacological cardioversion, all patients should receive anticoagulation therapy, also in case of $\mathrm{AF}<48$ hours, and irrespective of risk factors for stroke

- Post-cardioversion anticoagulation is mandated for 4 weeks in all patients, irrespective of risk factors for stroke

- The variety of antithrombotic regimens in the setting of catheter ablation calls for local protocols on periprocedural management, with most evidence for uninterrupted regimens

cate preprocedural anticoagulation for all patients with $\mathrm{AF}<48$ hours, regardless of stroke risk factors (Class IIaB) $[3,10]$. In the context of stroke rates of $0.5-1 \%$ in the month after cardioversion, a properly powered randomised controlled trial is not realistic. This is why registry data currently represent the best available evidence on the benefit of standard anticoagulation prior to the ECV $[13,14]$. Similar restrictions apply to the evidence used to change recommendations on the post-cardioversion antithrombotic regimen.

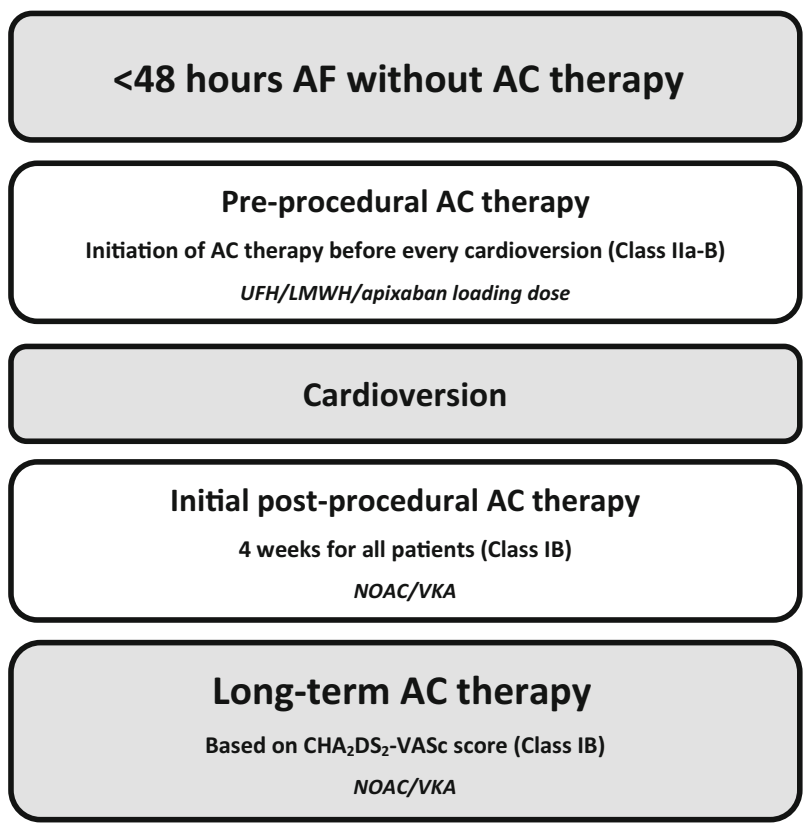

Note: Cardioversion may be performed without TEE (Class IIa-B)

\section{Postprocedural anticoagulation}

Another large registry, reporting outcome in 16,274 patients from Denmark, demonstrated that the risk of no anticoagulation therapy was similar for patients with a $\mathrm{CHA}_{2} \mathrm{DS}_{2}$ VASc score of 0-1 (odds ratio [OR] 2.21; 95\% confidence interval [CI] 0.79-6.77) and those with a $\mathrm{CHA}_{2} \mathrm{DS}_{2}$-VASc score of $\geq 2$ (OR 2.41; 95\% CI 1.46-3.95) [14]. Whereas previously the recommendation of a 4 -week anticoagulation strategy after ECV was restricted to patients with risk factors for stroke, these and other observations support the current advice to prescribe oral anticoagulation therapy after ECV in all patients [3, 10, 13-16]. First, the risk of stroke increases after cardioversion, not only for patients
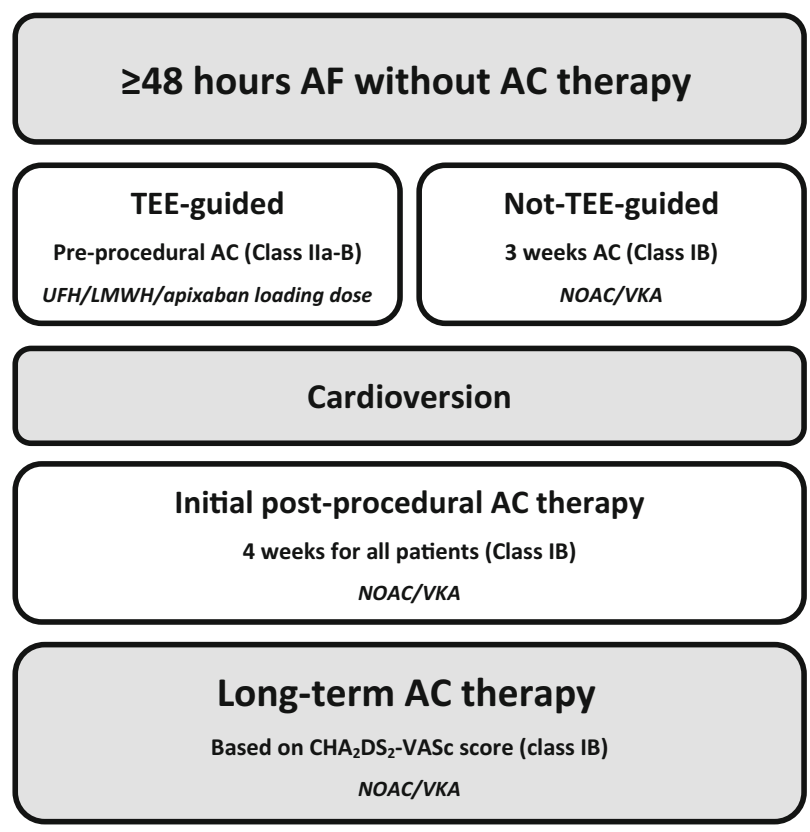

Note: If thrombus is found on TEE, start 3 weeks of AC therapy (Class I-C) and repeat TEE to ensure thrombus resolution (Class Ila-C)

Fig. 1 Pericardioversion anticoagulation: key recommendations in the ESC 2016 AF guidelines (ESC European Society of Cardiology, $A F$ atrial fibrillation, $A C$ anticoagulation, $U F H$ unfractionated heparin, $L M W H$ low-molecular-weight heparin, NOAC non-vitamin K oral anticoagulant, $V K A$ vitamin $\mathrm{K}$ antagonist, $\mathrm{CHA}_{2} \mathrm{DS}_{2}$-VASc score Congestive heart failure, Hypertension, Age $\geq 75$ [doubled], Diabetes, prior Stroke [doubled]_-Vascular disease, Age 65-74, Sex category, TEE transoesophageal echocardiography) 
Table 1 Cardioversions and thrombotic complications in patients with acute atrial fibrillation in the FinCV Study

\begin{tabular}{|c|c|c|c|c|c|c|}
\hline \multirow{3}{*}{$\begin{array}{l}\text { Patients without anticoagulation } \\
\text { therapy }\end{array}$} & \multicolumn{6}{|c|}{ Duration of atrial fibrillation } \\
\hline & \multicolumn{2}{|c|}{$<12$ hours $(n=2440)$} & \multicolumn{2}{|c|}{$12-24$ hours $(n=1840)$} & \multicolumn{2}{|c|}{$24-48$ hours $(n=836)$} \\
\hline & $N(\%)$ & $95 \% \mathrm{CI}$ & $N(\%)$ & $95 \% \mathrm{CI}$ & $N(\%)$ & $95 \% \mathrm{CI}$ \\
\hline Stroke/systemic embolism & $8(0.3)$ & $0.1-0.6 \%$ & $21(1.1)$ & $0.7-1.6 \%$ & $9(1.1)$ & $0.4-1.8 \%$ \\
\hline
\end{tabular}

CI confidence interval

with a high, but also for patients with a very low $\mathrm{CHA}_{2} \mathrm{DS}_{2}-$ VASc score. For patients with a $\mathrm{CHA}_{2} \mathrm{DS}_{2}$-VASc score of $0-1$, with a yearly stroke risk of up to $1.3 \%$, the observed 30-day stroke rate post-cardioversion is about $0.5 \%$. In the context of the estimated 1-year stroke risk, this represents a marked increase [6,10]. Even in patients with AF lasting less than 48 hours, the 30-day stroke risk has been reported to be considerably higher than the estimated 1-month risk as would be expected based upon the annual stroke risk [3, 13]. In short, when a patient is referred for $\mathrm{ECV}$, we predispose the patient to a higher risk of stroke, even when using the recommended postprocedural anticoagulation $[6,13]$. About half of these strokes occur within 2-5 days [12, 17, 18]. Importantly, the pathophysiological mechanism of the thrombo-embolic events is multifactorial, and even more complex than during the natural course of AF [15]. Preprocedural duration of AF, $\mathrm{CHA}_{2} \mathrm{DS}_{2}-\mathrm{VASc}$ score and parameters that indicate atrial function recovery have all been associated with the risk of thrombotic complications [11, 13, 15, 19]. Importantly, the effect on electrical atrial remodelling not only applies to electrical, but also to pharmacological cardioversion [19]. These findings support the revisions in the present guideline, that endorse post-cardioversion anticoagulation in all patients with $\mathrm{AF}<48$ hours, irrespective of risk factors (Class I-B). As for anticoagulation beyond the 4-week interval, no changes have been reported and the indication should be made based upon the $\mathrm{CHA}_{2} \mathrm{DS}_{2}$-VASc score (Class I-B).

\section{Unfractionated heparin (UFH), low-molecular-weight heparin (LMWH)}

Despite the focus on a stricter anticoagulation regimen, also in $\mathrm{AF}<48$ hours, little information is provided on the recommended dose of preprocedural heparin. In summary, most studies report for UFH a dose of $70-80 \mathrm{IU} / \mathrm{kg}$, with a total bolus of about 5000IU [20, 21]. The effect of UFH sets in quickly (within half an hour) and lasts for about 1-2 hours. As for enoxaparin, a subcutaneous dose of $1 \mathrm{mg} / \mathrm{kg}$ twice daily is most commonly reported; it has a slower onset of action (3-5 hours after subcutaneous injection) $[20,22$, 23]. Please note that a vitamin $\mathrm{K}$ antagonist (VKA) prescribed as a post-cardioversion anticoagulation strategy in anticoagulation-naive patients requires bridging with enoxaparin or UFH until the international normalised ratio (INR) has reached the target range. As stated before, ECV by itself creates a pro-thrombotic state with events often seen within the first 2-5 days and thus early, optimal anticoagulation is a prerequisite $[12,17,18]$.

\section{NOACs versus VKA}

Appreciating the low event rates in the setting of ECV, properly powered trials are a logistical challenge. Dabigatran was the first NOAC mentioned in the guidelines for the indication of ECV, based on a subgroup analysis from the RE-LY trial [24, 25]. Currently, subgroup analyses from all four large AF trials are available and several observational studies have been reported (Table 2; [24, 26-33]). In addition, randomised trials for rivaroxaban, edoxaban and apixaban have been completed [7-9]. Given their direct onset of the anticoagulant effect, NOACs are also promising drugs to substitute preprocedural heparin/LMWH. The only randomised trial that provides data on patients with $\mathrm{AF}<48$ hours is the EMANATE study, in which a subset of 342 patients received a loading dose of apixaban, administered at least two hours before cardioversion, the majority under guidance of transoesophageal echocardiography (TEE) [9]. Thus, most evidence of NOACs in the setting of cardioversion pertains to AF with a duration of $>$ 48 hours (Table 3 ). To expedite early cardioversion, there was a minimal pretreatment duration with rivaroxaban of 15 days, and about 3 days with edoxaban (Table 4; [7, 8]). For apixaban, a $10 \mathrm{mg}$ loading dose with subsequent cardioversion was possible ( 2 hours after intake), in other cases at least five regular doses of apixaban were required before cardioversion [9]. A meta-analysis on randomised data and information obtained from subgroup analyses demonstrates similar efficacy $(0.4 \%)$ and safety $(0.6 \%)$ between NOACs and VKA and thereby support the use of NOACs in the setting of ECV (Tables 5 and 6; [34]).

In summary, preprocedural anticoagulation for short duration $\mathrm{AF}$ is currently also recommended in patients without risk factors for stroke. Theoretically, the standard preprocedural administration of anticoagulation may carry a small, though somewhat higher risk of bleeding. With regard to post-cardioversion anticoagulation in all patients, this may have consequences as well, although the safety profile of NOACs is reassuring. 
Table 2 Post hoc analyses on NOAC and cardioversion

\begin{tabular}{lllll}
\hline Trial & RE-LY & ROCKET-AF & ARISTOTLE & ENGAGE AF-TIMI 48 \\
\hline NOAC & Dabigatran & Rivaroxaban & Apixaban & Edoxaban \\
Patients & $n=1270$ & $n=285$ & $n=540$ & $n=365^{*}$ \\
Number of CVs & 1983 & 375 & 743 & 632 \\
On randomised treatment & $80 \%$ & $87 \%$ & $84 \%$ & $100 \%$ \\
TEE-guided CVs & $21 \%$ & n/a & $27 \%$ & n/a \\
Follow-up duration & 30 days & 30 days & 30 days & 30 days \\
\hline
\end{tabular}

$A F$ atrial fibrillation, TIMI Thrombolysis in Myocardial Infarction, NOAC non-vitamin $\mathrm{K}$ oral anticoagulant, $C V s$ cardioversions, TEE transoesophageal echocardiography, $n / a$ not available

*including 111 patients on low-dose edoxaban $(30 \mathrm{mg} / 15 \mathrm{mg})$

Table 3 Randomised trials on NOAC and cardioversion

\begin{tabular}{llll}
\hline Trial & X-VeRT & EMANATE & ENSURE-AF \\
\hline Comparison & Rivaroxaban vs. VKA & Apixaban vs. heparin/warfarin & Edoxaban vs. enoxaparin/warfarin \\
Patients & $n=1504$ & $n=1500$ & $n=2199$ \\
AF duration & $\geq 48 \mathrm{~h}$ & $<48$ hours and $\geq 48$ hours ${ }^{\mathrm{a}}$ & $\geq 48 \mathrm{~h}$ \\
Treatment strata & Early or delayed & Imaging or no imaging & TEE or non-TEE \\
Stratum 1 & Early $(58 \%)$ & Imaging $(57 \%)^{\mathrm{b}}$ & TEE $(54 \%)$ \\
$\geq 3$ weeks OAC & $47 \%$ & $\mathrm{n} / \mathrm{a}$ & $\mathrm{n} / \mathrm{a}$ \\
TEE-guided CV & Rivaroxaban $67 \%$, VKA $65 \%$ & $100 \%{ }^{\mathrm{c}}$ & $100 \%$ \\
Stratum 2 & Delayed $(42 \%)$ & No imaging $(43 \%)^{\mathrm{b}}$ & Non-TEE $(46 \%)$ \\
$\geq 3$ weeks OAC & $100 \%$ & $\mathrm{n} / \mathrm{a}$ & $100 \%$ \\
TEE-guided CV & Rivaroxaban $8 \%$, VKA $14 \%$ & $0 \%$ & $0 \%$ \\
\hline
\end{tabular}

$A F$ atrial fibrillation, $C V$ cardioversion, $N O A C$ non-vitamin $\mathrm{K}$ oral anticoagulation, $O A C$ oral anticoagulation, $T E E$ transoesophageal echocardiography, $V K A$ vitamin $\mathrm{K}$ antagonist

a $2 / 3$ of patients had new onset $\mathrm{AF}$

${ }^{\mathrm{b}}$ optional loading dose of $10 \mathrm{mg}$ apixaban $\geq 2 \mathrm{~h}$ before cardioversion

'TEE or computed tomography

Table 4 Anticoagulation strategies in randomised trials on NOAC and cardioversion

\begin{tabular}{|c|c|c|c|c|c|c|}
\hline Trial & \multicolumn{2}{|l|}{$\mathrm{X}-\mathrm{VeRT}$} & \multicolumn{2}{|l|}{ EMANATE } & \multicolumn{2}{|c|}{ ENSURE-AF } \\
\hline Comparison & Rivaroxal & vs. VKA & Apixaban vs. hep & $\mathrm{n} /$ warfarin & Edoxabar & xaparin/warfarin \\
\hline Treatment strategy & TEE & No TEE & Imaging & No imaging & TEE & No TEE \\
\hline Pre-procedural anticoagulation & $1-5$ days & $3-8$ weeks & $\begin{array}{l}\sim 60 \% \text { 3-4 days }{ }^{\mathrm{a}} \text {; } \\
\sim 40 \% \text { 2-3 weeks }\end{array}$ & $\begin{array}{l}\sim 25 \% 3-4 \text { days }^{\mathrm{a}} \text {; } \\
\sim 75 \% 4 \text { weeks }\end{array}$ & $\leq 3$ days & $\geq 3$ weeks \\
\hline
\end{tabular}

Post-procedural anticoagulation

42 days

30 days

28 days

$A F$ atrial fibrillation, $V K A$ vitamin $\mathrm{K}$ antagonist, TEE transoesophageal echocardiography, NOAC non-vitamin-K anticoagulant

aloading dose of apixaban

Importantly, for early, immediate cardioversions (for example, in case of haemodynamic instability) in anticoagulation-naive patients UFH intravenously provides an immediate anticoagulant effect. The only NOAC tested with early cardioversions is apixaban (loading dose $10 \mathrm{mg}$ ), but this still requires a 2-hour interval before to proceed. The other NOACs were used for at least one day before ECV was performed. As for elective cardioversions, a 3-week pretreatment period, with at least for 4 weeks of post-treatment has been studied with all NOACs.

\section{Compliance}

In contrast to the situation with VKA, there is no objective tool to monitor the quality of anticoagulation of the preprocedural period with NOACs. Compliance is therefore of the utmost importance, and verification of compliance on the day of the ECV is crucial. Observations in patients treated with VKA have demonstrated that complications are much more frequent in those with a suboptimal intensity at the day of the procedure [18]. Although the randomised trials with NOACs on ECV do not provide very detailed definitions on preprocedural compliance, we advocate that none of the doses should be omitted in the three days before the 
Table 5 Efficacy and safety outcomes in post hoc analyses on NOAC and cardioversion

\begin{tabular}{lllll}
\hline Trial & RE-LY & ROCKET-AF & ARISTOTLE & ENGAGE AF-TIMI 48 \\
\hline Comparison & Dabigatran vs. warfarin & Rivaroxaban vs. warfarin & Apixaban vs. warfarin & Edoxaban vs. warfarin \\
SSE $^{\mathrm{a}}$ & $11(0.6 \%)$ & $2(0.7 \%)$ & $0(0 \%)$ & $0(0 \%)$ \\
NOAC vs. VKA & $7(0.5 \%)$ vs. $4(0.6 \%)$ & $\mathrm{n} / \mathrm{a}$ & - & - \\
Major bleeding $^{\mathrm{a}}$ & $19(1.0 \%)$ & $\mathrm{n} / \mathrm{a}$ & $2(0.2 \%)$ & $0(0 \%)$ \\
NOAC vs. VKA & $15(1.1 \%)$ vs. $4(0.6 \%)$ & $\mathrm{n} / \mathrm{a}$ & $1(0.3 \%)$ vs. $1(0.2 \%)$ & - \\
\hline
\end{tabular}

$A F$ atrial fibrillation, $S S E$ stroke or systemic embolism, $n / a$ not available, NOAC non-vitamin-K oral anticoagulant, TIMI Thrombolysis in Myocardial Infarction, $V K A$ vitamin $\mathrm{K}$ antagonist

${ }^{\mathrm{a} A f t e r} 30$ days

Table 6 Efficacy and safety outcomes in randomised trials on NOAC and cardioversion

\begin{tabular}{|c|c|c|c|}
\hline Trial & $\mathrm{X}$-VeRT & EMANATE & ENSURE-AF \\
\hline Comparison & Rivaroxaban vs. VKA & Apixaban vs. heparin/warfarin & Edoxaban vs. enoxaparin/warfarin \\
\hline Primary efficacy endpoint ${ }^{\mathrm{a}}$ & $10(0.7 \%)$ & $6(0.4 \%)$ & $16(0.7 \%)$ \\
\hline NOAC vs. VKA & $5(0.5 \%)$ vs. $5(1.0 \%)$ & $0(0 \%)$ vs. $6(0.8 \%)$ & $5(0.5 \%)$ vs. $11(1.0 \%)$ \\
\hline Primary safety endpoint ${ }^{\mathrm{b}}$ & $10(0.7 \%)$ & $33(2.2 \%)$ & $27(1.2 \%)$ \\
\hline NOAC vs. VKA & $6(0.6 \%)$ vs. $4(0.8 \%)$ & $14(1.9 \%)$ vs. $19(2.5 \%)$ & $16(1.5 \%)$ vs. $11(1.0 \%)$ \\
\hline
\end{tabular}

$A F$ atrial fibrillation, $N O A C$ non-vitamin-K oral anticoagulant, $V K A$ vitamin $\mathrm{K}$ antagonist

${ }^{a}$ X-VeRT: stroke/systemic embolism (SSE), transient ischaemic attack (TIA), myocardial infarction (MI) or cardiovascular (CV) death; EMANATE: SSE; ENSURE-AF: SSE, MI or CV death

${ }^{b}$ X-VeRT: International Society on Thrombosis and Haemostasis bleeding scale (ISTH) major bleeding; EMANATE and ENSURE-AF: major and clinically relevant non-major bleeding

procedure. The general rule that compliance is considered sufficient in case of an intake of $80 \%$ or more seems too arbitrary in the setting of cardioversion, as it does not cover the scenario that two missed doses in the three days before the procedure may still render a more than $80 \%$ intake [35].

\section{Daily practice}

From surveys in Europe, including the RHYTHM-AF registry, we have learned that our current practice of ECV has improved over the years $[1,36,37]$. The prescription of preprocedural antiarrhythmic drugs, which has been shown to improve success rates, still deserves attention [3]. Many centres experience an increase in the number of cardioversions per year, and at least $40 \%$ of the interviewed centres started implementing periprocedural use of NOACs [37]. Overall, estimates are that at present at least $25 \%$ of all cardioversions are performed on NOACs [37]. Little information is available on approaches that will ensure and control medication adherence, but as of yet the registry data on thrombo-embolic events are reassuringly low [29-32].

As for AF $>48$ hours, the majority of centres $(\sim 70 \%)$ followed the mandatory 3 -week period of oral anticoagulation prior to elective cardioversion, and in the other centres a TEE-guided approach was adopted [37]. In this survey, performed before the latest guideline update, about a third of the centres already had a protocol with 4 weeks of postcardioversion anticoagulation for $\mathrm{AF}<48$ hours, even when the embolic risk was low [37].
These surveys provide very informative insights into current developments and the areas in which we can still improve with regard to both antiarrhythmic and antithrombotic management in the setting of ECV.

\section{Ablation therapy for atrial fibrillation}

Over the past years, invasive treatment of atrial arrhythmias has evolved, and most of the current evidence on antithrombotic therapy has been collected in the setting of cryoballoon and radiofrequency ablation therapy [38]. Both thrombotic complications and bleeding events are events related to the specific technique of the procedure, the underlying disease and periprocedural antithrombotic management.

In addition to the induced tissue damage, temperature changes with application of cryoenergy and radiofrequency also affect the thrombotic state. Moreover, in some cases, $\mathrm{AF}$ is converted into sinus rhythm during the procedure, which is an additional mechanism that may contribute to a higher thrombotic risk, as described earlier for cardioversions [39].

The abovementioned conditions underscore why optimal procedural anticoagulation is a prerequisite. At present, this is recommended by use of heparin and a target activated clotting time of $>300 \mathrm{sec}$, although data to confirm this target are lacking. To minimise the presence of intra-atrial thrombus prior to the procedure, a period of 4 weeks of preprocedural anticoagulation has been advocated, in line with 
the recommendations for oral anticoagulation for ECV [39]. Historically, acenocoumarol and phenprocoumon were the drugs of choice for preprocedural anticoagulation in the Netherlands, which were discontinued prior to the procedure. Bridging with LMWH was performed before and after the ablation, followed by resumption of VKA. In the past few years, uninterrupted anticoagulation has become the standard, and the first randomised evidence for periprocedural use of NOACs has been published [40-42].

In addition to a brief review of the evidence, we will highlight a European survey on current practice, and provide some practical guidance for optimal periprocedural anticoagulation care [43].

\section{Peri-ablation anticoagulation-the evidence}

\section{VKA}

After the completion of the COMPARE trial, uninterrupted use of VKA has become the standard, and bridging is not recommended anymore [40]. In this randomised study with over 1500 patients continuous use of VKA (INR 2.0-3.0) was associated with a significantly lower rate of thromboembolic events, and a 40-50\% reduction in major bleeding as compared with a strategy with LMWH bridging [40]. Despite this improvement, the use of VKA has been associated with a disadvantage; the intensity of anticoagulation tends to fluctuate, and in about $20 \%$ of patients the INR is not in the target range prior to the procedure.

\section{NOAC}

After the introduction of NOACs many observational studies have been conducted to describe experiences with these new agents in the setting of ablation for AF [44]. Especially the timing of holding and resumption of these new drugs was largely unknown. Given its direct onset of effect, timing of resumption may affect postprocedural bleeding. Detailed analyses of the observational studies have confirmed this, showing that the studies with the most marked rates of bleeding and tamponade were characterised by a very short NOAC-free interval. Appreciating that heparin is administered during the procedure, and the initially unknown effects of combined heparin and NOAC use, many of the first studies used a regimen of interruption of NOAC. The first observational evidence suggested numerically, though not statistically significantly higher rates of thrombo-embolic events for interrupted NOAC regimens, with similar or somewhat lower bleeding rates when compared with warfarin regimens [45]. Meta-analyses on all observational data comparing interrupted regimens of NOAC versus VKA showed no difference in thrombo-embolic events and bleeding [45]. With increasing familiarity with the new drugs, uninter- rupted periprocedural NOAC use has become a more accepted strategy. For a fair comparison between NOAC and VKA with regard to bleeding complications, both should be used in a setting of uninterrupted anticoagulation. This is in contrast to a recent, randomised Asian study that showed lower bleeding rates in case of interrupted NOAC use versus continued use of VKA [46]. The available observational studies that compared continued periprocedural use of NOACs with uninterrupted VKA reported similar efficacy and safety [45]. Randomised controlled trials were eagerly awaited. Based on the reported rates of periprocedural stroke in the meta-analyses, it should be acknowledged that - a priori-these trials were not powered to address this endpoint. Therefore, the emphasis shifted towards 'safety and feasibility', although it should be appreciated that some of the trials have been criticised for the design and power to address major bleeding as well. As of yet, two trials are currently recruiting. First, the ELIMINATE (A prospective, randomised, open-label, blinded endpoint evaluation parallel group study comparing edoxaban versus VKA in subjects undergoing catheter ablation of nonvalvular atrial fibrillation; NCT02942576) study in which uninterrupted anticoagulation with edoxaban versus VKA is the topic of interest. The trial will not be performed in the Netherlands and France due to alleged issues with the study design. However, calculations based upon the RECIRCUIT trial demonstrate that for the endpoint bleeding, power should not be an issue. The AXAFA trial has addressed the same study question for apixaban [9, 47]. The first randomised, though somewhat small study $(n=248)$ of NOACs versus VKA was VENTURE-AF, in which rivaroxaban $20 \mathrm{mg}$ once daily resulted in similar bleeding rates as uninterrupted VKA [41]. The largest trial so far $(n=635)$ has been performed with dabigatran, where patients in the RE-CIRCUIT trial had significantly higher bleeding rates on VKA than on uninterrupted NOAC [42].

Just recently, an updated meta-analysis on 12 studies, with in total 4962 patients, suggested similar rates of thrombo-embolism (NOAC $0.08 \%$ vs. VKA $0.16 \%$; OR 0.66 , 95\% CI $0.19-2.30)$ and demonstrated significantly lower bleeding rates (NOAC $0.9 \%$ vs. VKA $2.0 \%$; OR $0.50,95 \%$ CI $0.30-0.84, p<0.01$ ) when uninterrupted use of NOACs was compared with uninterrupted use of VKA, in observational and randomised trials taken together [44]. The first evidence on interrupted NOAC regimens looks promising with regard to safety, but additional information with regard to the embolic protection is warranted [48].

\section{Peri-ablation anticoagulation-daily practice}

In a 2014 European Heart Survey on periprocedural anticoagulation about 50-60\% reported to use VKA prior to 
the procedure, with uninterrupted periprocedural VKA in $80 \%$. In about $10 \%$ of procedures interrupted VKA with bridging was the periprocedural regimen. This latter strategy was less frequently observed in high volume centres and university hospitals [43].

Notably, a strategy of uninterrupted VKA has the logistic drawback that about $20 \%$ of patients will not have adequate INR control in the weeks prior to the procedure. Data on procedures performed in case of inadequate INR control prior to the procedure is scarce, although it might be considered in patients with a low risk for stroke. Procedural anticoagulation is secured with heparin administration that is driven by activated clotting time, but postprocedural anticoagulation coverage may be suboptimal. Appreciating the elective nature of the procedure, primarily focused on comfort and complaints and not on prognosis, inadequate INR control before the procedure may therefore result in postponement of the procedure.

In analogy to the planning of cardioversions for AF, NOACs have the advantage of predictable planning of the procedures. Currently, $30 \%$ of patients in Europe now receive a NOAC prior to the ablation. In half, the use is uninterrupted or just briefly interrupted. Strikingly, in the other half of cases an interruption of more than 2 days is reported, which results in suboptimal periprocedural coverage. This shows that more information is needed to guide the clinical approach of periprocedural use of NOACs [43].

At present, most of the available randomised and observational studies apply to dabigatran, followed by studies on experiences with rivaroxaban [48]. Uninterrupted regimens guarantee continuous anticoagulation, providing optimal protection against thrombo-embolic events. As for dabigatran, taken twice daily, the protocol of the RE-CIRCUIT study stated that the morning dose was not to be omitted before the ablation. Resumption after the procedure was at the same day, with a minimum of 3 hours after sheath removal [42]. For the once daily regimen of rivaroxaban, evening dosing was recommended and the factor Xa inhibitor could be re-initiated 6 hours after haemostasis was established [41].

These differences between these approaches demonstrate how important it is to define local protocols, both pre-ablation and post-ablation. If a regimen of uninterrupted anticoagulation is preferred, the NOACs with a once daily administration deserve special attention with regard to the time of administration (evening dosing). A short preprocedural interval of NOAC interruption will not cause much harm, given the often low $\mathrm{CHA}_{2} \mathrm{DS}_{2}$-VASc score of most patients undergoing ablation therapy [38, 42]. However, intervals of $>2$ days as reported in the recent survey should be avoided, not only in patients in AF prior to the procedure, but also in other patients to ensure a postprocedural antithrombotic coverage in the first few hours. Another im- portant aspect concerns compliance. Whereas INR control provided an indication of the quality of preprocedural anticoagulation with VKA, in the NOAC era the patient is our only source. It should be noted that prior to the procedure the risk of thrombo-embolic events is extremely low for many patients in the target population, which may explain that about $10 \%$ of patients in the survey did not have any anticoagulation prior to the procedure [43]. Importantly, the proper level of anticoagulation during the procedure can always be achieved by administering heparin. However, to prevent procedure-related thrombo-embolic events it is of the utmost importance to keep the NOAC free interval after the procedure as short as possible. On the other hand, the interval should be long enough to ensure haemostasis at puncture sites.

In summary, there is a lot of experience and randomised evidence on strategies of uninterrupted use of VKA, with clear superiority in terms of major bleeding when compared to interrupted VKA and heparin bridging. In addition, thrombo-embolic events on the uninterrupted regimen of VKA are very low. In daily practice, the implementation of uninterrupted VKA seems satisfactory.

In contrast, centres using NOACs often fail to follow the approach as tested in the limited available randomised trials, which calls for attention to improve our daily practice. With an uninterrupted regimen, evening dosing is recommended for rivaroxaban. In case of dabigatran the morning dose does not need to be skipped. Resumption after the procedure is recommended 3-5 hours after haemostasis has been established. As far as the post-ablation period is concerned, at least 2 months of postprocedural anticoagulation is warranted. Long-term anticoagulation should be continued as per the original indication for subjects with risk factors for stroke, based on the $\mathrm{CHA}_{2} \mathrm{DS}_{2}$-VASc score. Data on the other NOACs for the indication of ablation are eagerly awaited, as well as additional evidence for interrupted regimens.

Conflict of interest S.P.G. van Vugt has received consulting fees/ honorariums from Bayer, Boehringer Ingelheim, Bristol-MeyersSquibb/Pfizer and Daiichi Sankyo and M.A. Brouwer has received consulting fees/honorariums from AstraZeneca, Bayer, Boehringer Ingelheim, Bristol-Meyers-Squibb/Pfizer and Daiichi Sankyo.

Open Access This article is distributed under the terms of the Creative Commons Attribution 4.0 International License (http:// creativecommons.org/licenses/by/4.0/), which permits unrestricted use, distribution, and reproduction in any medium, provided you give appropriate credit to the original author(s) and the source, provide a link to the Creative Commons license, and indicate if changes were made. 


\section{References}

1. Crijns HJ, Weijs B, Fairley AM, et al. Contemporary real life cardioversion of atrial fibrillation: results from the multinational RHYTHM-AF study. Int J Cardiol. 2014;172(3):588-94.

2. Bellone A, Etteri M, Vettorello M, et al. Cardioversion of acute atrial fibrillation in the emergency department: a prospective randomised trial. Emerg Med J. 2012;29(3):188-91.

3. Kirchhof P, Benussi S, Kotecha D, et al. 2016 ESC Guidelines for the management of atrial fibrillation developed in collaboration with EACTS. Eur Heart J. 2016;37(38):2893-962.

4. Letelier LM, Udol K, Ena J, et al. Effectiveness of amiodarone for conversion of atrial fibrillation to sinus rhythm: a meta-analysis. Arch Intern Med. 2003;163(7):777-85.

5. Bash LD, Buono JL, Davies GM, et al. Systematic review and metaanalysis of the efficacy of cardioversion by vernakalant and comparators in patients with atrial fibrillation. Cardiovasc Drugs Ther. 2012;26(2):167-79.

6. Airaksinen KJ. Cardioversion of atrial fibrillation and oral anticoagulation. J Atr Fibrillation. 2015;8(3): 1260.

7. Cappato R, Ezekowitz MD, Klein AL, et al. Rivaroxaban vs. vitamin $\mathrm{K}$ antagonists for cardioversion in atrial fibrillation. Eur Heart J. 2014;35(47):3346-55.

8. Goette A, Merino JL, Ezekowitz MD, et al. Edoxaban versus enoxaparin-warfarin in patients undergoing cardioversion of atrial fibrillation (ENSURE-AF): a randomised, open-label, phase $3 \mathrm{~b}$ trial. Lancet. 2016;388(10055):1995-2003.

9. Ezekowitz MD, Pollack CV, Halperin JL, et al. Apixaban compared to heparin/vitamin $\mathrm{K}$ antagonist in patients with atrial fibrillation scheduled for cardioversion: the EMANATE trial. Eur Heart J 2018. Apr 6 (epub ahead of print).

10. Camm AJ, Kirchhof P, Lip GY, et al. Guidelines for the management of atrial fibrillation: the Task Force for the Management of Atrial Fibrillation of the European Society of Cardiology (ESC). Eur Heart J. 2010;31(19):2369-429.

11. Nuotio I, Hartikainen JE, Gronberg T, et al. Time to cardioversion for acute atrial fibrillation and thromboembolic complications. JAMA. 2014;312(6):647-9.

12. Airaksinen KE, Gronberg T, Nuotio I, et al. Thromboembolic complications after cardioversion of acute atrial fibrillation: the FinCV (Finnish CardioVersion) study. J Am Coll Cardiol. 2013;62(13): 1187-92.

13. Gronberg T, Hartikainen JE, Nuotio I, et al. Anticoagulation, CHA2DS2VAsc score, and thromboembolic risk of cardioversion of acute atrial fibrillation (from the FinCV study). Am J Cardiol. 2016;117(8):1294-8.

14. Hansen ML, Jepsen RM, Olesen JB, et al. Thromboembolic risk in 16274 atrial fibrillation patients undergoing direct current cardioversion with and without oral anticoagulant therapy. Europace. 2015;17(1):18-23.

15. Fatkin D, Kuchar DL, Thorburn CW, et al. Transesophageal echocardiography before and during direct current cardioversion of atrial fibrillation: evidence for "atrial stunning" as a mechanism of thromboembolic complications. J Am Coll Cardiol. 1994;23(2):307-16.

16. Lip GY, Gitt AK, Le Heuzey JY, et al. Overtreatment and undertreatment with anticoagulation in relation to cardioversion of atrial fibrillation (the RHYTHM-AF study). Am J Cardiol. 2014;113(3):480-4.

17. Berger M, Schweitzer P. Timing of thromboembolic events after electrical cardioversion of atrial fibrillation or flutter: a retrospective analysis. Am J Cardiol. 1998;82(12):1545-7.

18. Gallagher MM, Hennessy BJ, Edvardsson N, et al. Embolic complications of direct current cardioversion of atrial arrhythmias: as- sociation with low intensity of anticoagulation at the time of cardioversion. J Am Coll Cardiol. 2002;40(5):926-33.

19. Khan IA. Atrial stunning: basics and clinical considerations. Int $\mathbf{J}$ Cardiol. 2003;92(2-3):113-28.

20. Stellbrink C, Nixdorff U, Hofmann T, et al. Safety and efficacy of enoxaparin compared with unfractionated heparin and oral anticoagulants for prevention of thromboembolic complications in cardioversion of nonvalvular atrial fibrillation: the Anticoagulation in Cardioversion using Enoxaparin (ACE) trial. Circulation. 2004;109(8):997-1003.

21. Cohen A, Stellbrink C, Le Heuzey JY, et al. SAfety of fondaparinux in transoesophageal echocardiography-guided electric cardioversion of Atrial Fibrillation (SAFE-AF) study: a pilot study. Arch Cardiovasc Dis. 2015;108(2):122-31.

22. Klein AL, Jasper SE, Katz WE, et al. The use of enoxaparin compared with unfractionated heparin for short-term antithrombotic therapy in atrial fibrillation patients undergoing transoesophageal echocardiography-guided cardioversion: assessment of Cardioversion Using Transoesophageal Echocardiography (ACUTE) II randomized multicentre study. Eur Heart J. 2006;27(23):2858-65.

23. Garcia DA, Baglin TP, Weitz JI, et al. Parenteral anticoagulants: Antithrombotic Therapy and Prevention of Thrombosis, 9th ed: American College of Chest Physicians Evidence-Based Clinical Practice Guidelines. Chest. 2012;141(2 Suppl):e24S-e43S.

24. Nagarakanti R, Ezekowitz MD, Oldgren J, et al. Dabigatran versus warfarin in patients with atrial fibrillation: an analysis of patients undergoing cardioversion. Circulation. 2011;123(2):131-6.

25. Camm AJ, Lip GY, De Caterina R, et al. 2012 focused update of the ESC Guidelines for the management of atrial fibrillation: an update of the 2010 ESC Guidelines for the management of atrial fibrillation-developed with the special contribution of the European Heart Rhythm Association. Europace. 2012;14(10):1385-413.

26. Piccini JP, Stevens SR, Lokhnygina Y, et al. Outcomes after cardioversion and atrial fibrillation ablation in patients treated with rivaroxaban and warfarin in the ROCKET AF trial. J Am Coll Cardiol. 2013;61(19): 1998-2006.

27. Flaker G, Lopes RD, Al-Khatib SM, et al. Efficacy and safety of apixaban in patients after cardioversion for atrial fibrillation: insights from the ARISTOTLE Trial (Apixaban for Reduction in Stroke and Other Thromboembolic Events in Atrial Fibrillation). J Am Coll Cardiology. 2014;63(11):1082-7.

28. Plitt A, Ezekowitz MD, De Caterina R, et al. Cardioversion of Atrial Fibrillation in ENGAGE AF-TIMI 48. Clin Cardiol. 2016;39(6):345-6.

29. Kochhauser S, Khaykin Y, Beardsall J, et al. Comparison of outcomes after cardioversion or atrial fibrillation ablation in patients with differing periprocedural anticoagulation regimens. Can J Cardiol. 2014;30(12):1541-6.

30. Coleman CM, Khalaf S, Mould S, et al. Novel oral anticoagulants for DC cardioversion procedures: utilization and clinical outcomes compared with warfarin. Pacing Clin Electrophysiol. 2015;38(6):731-7.

31. Pallisgaard JL, Lindhardt TB, Hansen ML, et al. Cardioversion and risk of adverse events with dabigatran versus warfarin-a nationwide cohort study. PLoS ONE. 2015;10(10):e141377.

32. Frederiksen AS, Albertsen AE, Christesen AMS, Vinter N, Frost L, Moller DS. Cardioversion of atrial fibrillation in a real-world setting: non-vitamin $\mathrm{K}$ antagonist oral anticoagulants ensure a fast and safe strategy compared to warfarin. Europace. 2017; https://doi. org/10.1093/europace/eux188.

33. Itainen $\mathrm{S}$, Lehto $\mathrm{M}$, Vasankari $\mathrm{T}$, et al. Non-vitamin $\mathrm{K}$ antagonist oral anticoagulants in atrial fibrillation patients undergoing elective cardioversion. Europace. 2018;20(4):565.

34. Renda G, Ricci F, De Caterina R. Non-vitamin K antagonist oral anticoagulants for cardioversion in atrial fibrillation: an updated metaanalysis. Am J Med. 2017;130(4):457-61. 
35. Raval AN, Cigarroa JE, Chung MK, et al. Management of patients on non-vitamin $\mathrm{K}$ antagonist oral anticoagulants in the acute care and periprocedural setting: a scientific statement from the American heart association. Circulation. 2017;135(10):e604-e33.

36. Pisters R, Nieuwlaat R, Prins MH, et al. Clinical correlates of immediate success and outcome at 1-year follow-up of real-world cardioversion of atrial fibrillation: the Euro Heart Survey. Europace. 2012;14(5):666-74.

37. Hernandez-Madrid A, Svendsen JH, Lip GY, et al. Cardioversion for atrial fibrillation in current European practice: results of the European Heart Rhythm Association survey. Europace. 2013;15(6):915-8.

38. Kuck KH, Brugada J, Furnkranz A, et al. Cryoballoon or radiofrequency ablation for paroxysmal atrial fibrillation. N Engl J Med. 2016;374(23):2235-45.

39. Calkins H, Hindricks G, Cappato R, et al. 2017 HRS/EHRA/ECAS/ APHRS/SOLAECE expert consensus statement on catheter and surgical ablation of atrial fibrillation. Europace. 2018;20(1):e1-e160.

40. Di Biase L, Burkhardt JD, Santangeli P, et al. Periprocedural stroke and bleeding complications in patients undergoing catheter ablation of atrial fibrillation with different anticoagulation management: results from the Role of Coumadin in Preventing Thromboembolism in Atrial Fibrillation (AF) Patients Undergoing Catheter Ablation (COMPARE) randomized trial. Circulation. 2014;129(25):2638-44.

41. Cappato R, Marchlinski FE, Hohnloser SH, et al. Uninterrupted rivaroxaban vs. uninterrupted vitamin $\mathrm{K}$ antagonists for catheter ablation in non-valvular atrial fibrillation. Eur Heart J. 2015;36(28): 1805-11.

42. Calkins H, Willems S, Gerstenfeld EP, et al. Uninterrupted dabigatran versus warfarin for ablation in atrial fibrillation. N Engl J Med. 2017;376(17):1627-36.
43. Potpara TS, Larsen TB, Deharo JC, et al. Oral anticoagulant therapy for stroke prevention in patients with atrial fibrillation undergoing ablation: results from the First European Snapshot Survey on Procedural Routines for Atrial Fibrillation Ablation (ESS-PRAFA). Europace. 2015;17(6):986-93.

44. Cardoso R, Knijnik L, Bhonsale A, et al. An updated meta-analysis of novel oral anticoagulants versus vitamin $\mathrm{K}$ antagonists for uninterrupted anticoagulation in atrial fibrillation catheter ablation. Heart Rhythm. 2018;15(1):107-15.

45. Wu S, Yang YM, Zhu J, et al. Meta-analysis of efficacy and safety of new oral anticoagulants compared with uninterrupted vitamin $\mathrm{K}$ antagonists in patients undergoing catheter ablation for atrial fibrillation. Am J Cardiol. 2016;117(6):926-34.

46. Goya M, Nogami A, Hirao K, et al. Ablation perioperative dabigatran in use envisioning in Japan: the ABRIDGE-J study design. J Cardiol. 2016;68(3):236-40.

47. Di Biase L, Callans D, Haeusler KG, et al. Rationale and design of AXAFA-AFNET 5: an investigator-initiated, randomized, open, blinded outcome assessment, multi-centre trial to comparing continuous apixaban to vitamin $\mathrm{K}$ antagonists in patients undergoing atrial fibrillation catheter ablation. Europace. 2017;19(1):132-8.

48. Zhao Y, Yang Y, Tang X, et al. New oral anticoagulants compared to warfarin for perioperative anticoagulation in patients undergoing atrial fibrillation catheter ablation: a meta-analysis of continuous or interrupted new oral anticoagulants during ablation compared to interrupted or continuous warfarin. J Interv Card Electrophysiol. 2017;48(3):267-82.

49. Ezekowitz MD, Pollack CV, Sanders P, et al. Apixaban compared with parenteral heparin and/or vitamin $\mathrm{K}$ antagonist in patients with nonvalvular atrial fibrillation undergoing cardioversion: rationale and design of the EMANATE trial. Am Heart J. 2016;179:59-68. 